EPJ Web of Conferences 72, 00020 (2014)

DOI: $10.1051 /$ epjconf / 20147200020

(C) Owned by the authors, published by EDP Sciences, 2014

\title{
Exotic Hadron Spectroscopy
}

\author{
Alessandro Pilloni ${ }^{1, a}$ \\ ${ }^{1}$ Dipartimento di Fisica and INFN, "Sapienza" Università di Roma \\ P.le Aldo Moro 2 - I-00185 Roma (Italy)
}

\begin{abstract}
Since ten years ago experiments have been observing a host of exotic states decaying into heavy quarkonia. The interpretation of most of them still remains uncertain and, in some cases, controversial. Notwithstanding, a considerable progress has been made on the quality of the experimental information available and a number of ideas and models have been put forward to explain the observations. We will review the most promising theoretical interpretations of exotic states, in particular we will discuss the nature of the charmonium-like resonances in the region $3850-4050 \mathrm{MeV}$, and propose a new mechanism to explain prompt $X(3872)$ production cross section at hadron colliders. Finally, the phenomenology of doubly charmed states is commented.
\end{abstract}

\section{Introduction}

Heavy quarkonium sector has proved to be extremely useful for the understanding of QCD. In the limit $m_{Q} \rightarrow \infty$, the gauge field dynamics can be simplified in terms of effective potential models [1], or in terms of effective theories as NRQCD [2], which allow us to make accurate predictions about quarkonia spectra, production cross sections and decay rates. However, in the last ten years a lot of unexpected quarkonium-like resonances have been discovered. There are several hints that these states cannot fit standard heavy quarkonium interpretations, whence they were called $X, Y, Z$. A graphic summary of the situation in charm sector is reported in Fig. 1. Despite a lot of efforts, a comprehensive framework for a unified description of all these states is still missing. The actual identification of these states would represent a major progress in our understanding of strong interaction dynamics, and might imply the prediction of a large number of additional states that have not yet been observed.

The most likely possible states beyond ordinary mesons and baryons are:

- Molecules: bound states of two mesons, just like deuterium is the bound state of a proton and a neutron. The binding mechanism involves the exchange of a light meson $(\pi, \sigma)$ [3], or even a 0 -order contact interaction [4]. The mass of these states is expected to be near the sum of the two meson masses. In particular, the system would be stable if the binding energy is negative, so that the mass of the states would be set below meson threshold. The smaller is this binding energy, the larger is the spatial extension of the molecule, up to $10 \mathrm{fm}$ for $X(3872)$. The bound state should decay mainly into its constituents [5].
- Tetraquarks: a quark pair (diquark) in the $\overline{\mathbf{3}}_{c}$ (attractive) configuration, neutralizing its color with an antiquark pair [6,7]. A full nonet of states is predicted for each spin-parity, i.e. a large number of states are expected. There is no need for these states to be close to any threshold. Isospin-violating decay are predicted in a natural way. The lack of observations of predicted states constitutes the main drawback to this model.

- Hybrids: bound states of a quark-antiquark pair in the $\mathbf{8}_{c}$ configuration and of a number of constituent gluons. The lowest-lying state is expected to have quantum numbers $J^{P C}=0^{+-}$. Since a quarkonium state cannot have these quantum numbers, this is a unique signature for hybrids. An additional signature is the preference for

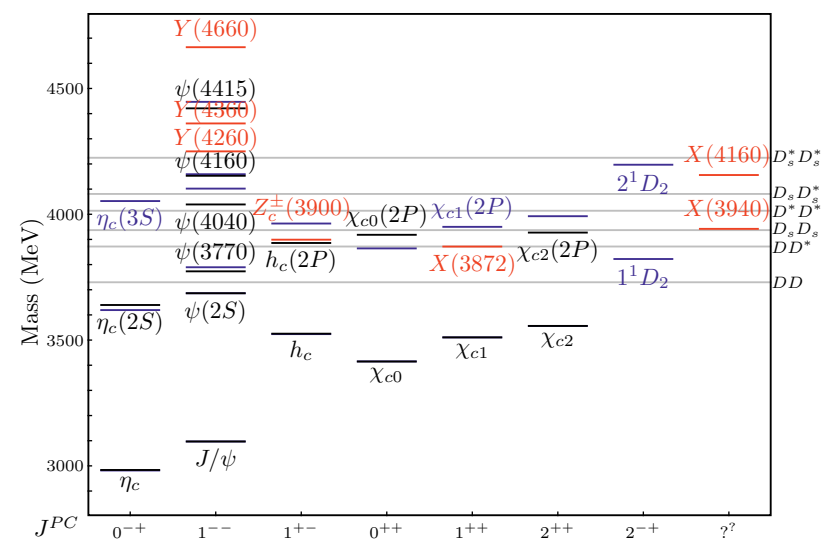

Figure 1. Charmonium sector. Black lines represent observed charmonium levels, blue lines represent predicted levels according to [1], red line are exotic states. The open charm threshold are reported on the right.

\footnotetext{
a e-mail: alessandro.pilloni@roma1.infn.it
} 


\begin{tabular}{c|c|c|c|c} 
State & Mass $(\mathrm{MeV})$ & Width $(\mathrm{MeV})$ & Decay channels & $\left(I^{G}\right) J^{P C}$ \\
\hline$X(3872)$ & $3871.68 \pm 0.17$ & $<1.2$ & see Ref. [14] & $\left(*^{*}\right) 1^{++}$ \\
\hline$Y(4260)$ & $4250 \pm 9$ & $108 \pm 12$ & see Ref. [14] & $\left(0^{-}\right) 1^{--}$ \\
\hline$Z_{c}(3900)$ & $3899 \pm 6$ & $46 \pm 22$ & $J / \psi \pi$ & likely $\left(1^{+}\right) 1^{+-}$ \\
$Z_{c}(3885)$ & $3883.9 \pm 4.5$ & $24.8 \pm 11.5$ & $D D^{*}$ & $\left(1^{+}\right) 1^{+-}$ \\
\hline$Z_{c}^{\prime}(4020)$ & $4022.9 \pm 2.8$ & $7.9 \pm 3.7$ & $h_{c} \pi$ & $\left(1^{+}\right) 1^{?-}$ \\
$Z_{c}^{\prime}(4025)$ & $4026.3 \pm 4.5$ & $24.8 \pm 9.5$ & $D^{*} D^{*}$ & $\left(1^{+}\right) 1^{+-}$ \\
\hline$Z_{b}(10610)$ & $10607.2 \pm 2.0$ & $18.4 \pm 2.4$ & $\Upsilon(n S) \pi, h_{b}(n P) \pi, B B^{*}$ & $\left(1^{+}\right) 1^{+-}$ \\
\hline$Z_{b}^{\prime}(10650)$ & $10652.2 \pm 1.5$ & $11.5 \pm 2.2$ & $\Upsilon(n S) \pi, h_{b}(n P) \pi, B^{*} B^{*}$ & $\left(1^{+}\right) 1^{+-}$
\end{tabular}

Table 1. Mass, width and quantum numbers of exotic states analysed. $I^{G}$ is undefined for $X(3872)$ because of isospin-violating decay.
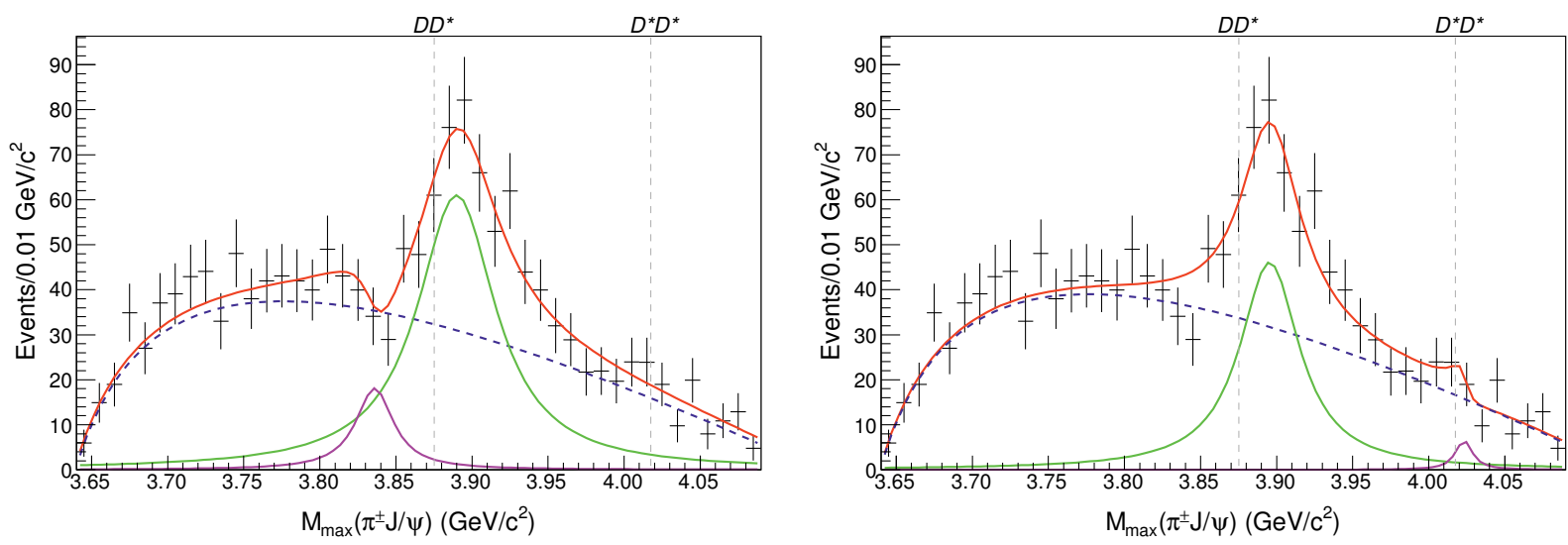

Figure 2. Result of the fit to the BES and Belle [10] data reported in Ref. [17], in the presence of a lighter resonance (left panel) or heavier resonance (right panel). The former fit has $\chi^{2} / \mathrm{DOF}=41 / 65$, whereas the latter fit has $\chi^{2} / \mathrm{DOF}=47 / 65$. Red curves: fit to data. Green curves: $Z_{c}(3900)$. Purple curves: additional resonances. Blue dashed curves: background.

a hybrid to decay into quarkonium and a state that can be produced by the excited gluons (e.g. $\pi^{+} \pi^{-}$pairs) [8].

- Hadrocharmonium (hadrobottomomium): a colorsinglet proto-quarkonium $Q \bar{Q}$ core is supposed to interact with a light quark cloud via a Van-der-Waals residual force [9]. Quantum numbers are the same of a pair quarkonium-light meson.

In this talk we would like to summarize the recent research on exotic hadrons by our group. In Sec. 2 we will talk about $Z_{c}$ states, and about its interpretation in terms of tetraquark model. In Sec. 3 we will show a possible comprehensive framework for most of known states in terms of Feshbach resonances. In Sec. 4 we will propose a new mechanism to explain huge $X(3872)$ prompt production cross section at hadron colliders. Finally, in Sec. 5 we propose a new tetraquark particle with peculiar quantum numbers and experimental signatures, whose existence can be checked in lattice simulations and at LHCb.

In this review we will rely on the set of best established states: in the charm sector we consider the $X(3872), Y(4260)$ (both seen by many experiments), and the charged states $Z_{c}(3900)$ [10]. Furthermore, we extend our analysis to some states recently announced by BES, i.e. $Z_{c}(3885)$ [11], $Z_{c}^{\prime}(4020)$ and $Z_{c}^{\prime}(4025)$ [12]. Finally, in the bottom sector we will consider $Z_{b}(10610)$ and
$Z_{b}^{\prime}$ (10650) [13], which look very much like the $b$-partners of $Z_{c}$ and $Z_{c}^{\prime}$. Mass, width and quantum numbers are reported in Tab. 1.

\section{Exotic resonances near $X(3872)$ and tetraquark model}

The $Z_{c}(3900)$ is a charged state seen by BES and Belle [10] as a resonance in $Y(4260) \rightarrow Z_{c}^{+}(3900) \pi^{-} \rightarrow J / \psi \pi^{+} \pi^{-1}$, likely carrying quantum number $J^{P C}=1^{+-}$. Some hints for the existence of an almost degenerate $Z_{c}^{0}$ neutral partner seem encouraging [15]. The $Z_{c}$ is described in several papers as a $D D^{*}$ molecule, the $I=1$ partner of the $X(3872)$ [16]. However, a real bound state must have negative binding energy, whereas $Z_{c}$ is about $25 \mathrm{MeV}$ above $D D^{*}$ threshold. Such above-threshold states should be considered a sort of virtual unstable states. However, the mechanism for which such resonances are pushed above threshold is still unclear.

In Ref. [7], assuming $X(3872)$ to be a $1^{++}$tetraquark, two $1^{+-}$states were predicted at $3882 \mathrm{MeV}$ and $3755 \mathrm{MeV}$. In Ref. [17] the $Z_{c}(3900)$ was identified as the $1^{+-}$heavier tetraquark state. The presence of a lighter state would support the tetraquark hypothesis, whereas molecular pic-

\footnotetext{
${ }^{1}$ Hereafter the charge-conjugated modes are understood.
} 
Lepton and Hadron Physics at Meson-Factories

\begin{tabular}{c|c|c|c|c|c} 
State & Hadro- $c \bar{c},-b \bar{b}$ & Open $c / b$ & $\Gamma(\mathrm{MeV})$ & $v(\mathrm{MeV})$ & $A\left(\mathrm{MeV}^{1 / 2}\right)$ \\
\hline$X(3872)$ & $J / \psi \rho^{0}$ & $D^{0} \bar{D}^{* 0}$ & 0 & 0 & - \\
\hline$Z_{c}(3900)$ & $\psi(3770) \pi$ & $D^{+} \bar{D}^{* 0}$ & $46 \pm 22$ & $23 \pm 6$ & $10 \pm 5$ \\
\hline$Z_{c}^{\prime}(4020)$ & $h_{c}(2 P) \pi_{P \text {-wave }}$ & $D^{*+} \bar{D}^{* 0}$ & $24.8 \pm 9.5$ & $9 \pm 4.5$ & $8 \pm 4$ \\
$Z_{c}^{\prime}(4025)$ & $h_{c}(2 P) \pi_{P \text {-wave }}$ & $D^{*+} \bar{D}^{* 0}$ & $7.9 \pm 3.7$ & $5.6 \pm 2.8$ & $3 \pm 2$ \\
\hline$Z_{b}(10610)$ & $\chi_{b 0}(1 P) \rho_{P \text {-wave }}$ & $B^{+} \bar{B}^{* 0}$ & $18.4 \pm 2.4$ & $3 \pm 2$ & $11 \pm 4$ \\
\hline$Z_{b}^{\prime}(10650)$ & $\chi_{b 0}(1 P) \rho_{P \text {-wave }}$ & $B^{*+} \bar{B}^{* 0}$ & $11.5 \pm 2.2$ & $1.8 \pm 1.5$ & $8 \pm 4$
\end{tabular}

Table 2. Exotic states in term of Feshbach resonances. The width is related to the detuning by $\Gamma=A \sqrt{v}$.

ture predicts a $D^{*} D^{*}$ bound state at $\sim 4020 \mathrm{MeV}$. Furthermore, it was noticed that the presence of a lighter state (even though at $\sim 3820 \mathrm{MeV}$ ) was not incompatible with data, whereas the presence of a peak at $4020 \mathrm{MeV}$ seemed unlikely (Fig. 2). All these facts are in favour of tetraquark hypothesis.

However, in the last months BES discovered two resonances close to the $D^{*} D^{*}$ threshold [12]: $Z_{c}^{\prime}(4025)$ was observed in the channel $e^{+} e^{-} \rightarrow Z_{c}^{\prime+}(4025) \pi^{-} \rightarrow D^{*+} \bar{D}^{* 0} \pi^{-}$, and $Z_{c}^{\prime}(4020)$ in the channel $e^{+} e^{-} \rightarrow Z_{c}^{\prime+}(4020) \pi^{-} \rightarrow$ $h_{c} \pi^{+} \pi^{-}$. The mass and width of these two resonances are compatible to each other respectively within $1 \sigma$ and $2 \sigma$. As for the quantum numbers, the former has $J^{P C}=1^{+-}$. On the other hand, the latter has $J^{P C}=1^{--}$if the $Z_{c}^{\prime}$ decays in $S$-wave ${ }^{2}$. If so, the neutral partner should be copiously produced in $e^{+} e^{-}$collisions. If we assume instead that the two $Z_{c}^{\prime}$ are the same particle, the latter must decay in $P$ wave to get the correct parity. In the following, we will choose the $1^{+-}$assignment and will identify the two resonances.

Finally, BES has recently announced [11] the discovery of a $Z_{c}(3885)$ in $e^{+} e^{-} \rightarrow Z_{c}^{+}(3885) \pi^{-} \rightarrow D^{*+} \bar{D}^{0} \pi^{-}$. Mass and width are compatible with $Z_{c}$ (3900) ones within $2 \sigma$ and $1 \sigma$.

These new discoveries challenge the tetraquark picture: the $Z_{c}^{\prime}$ cannot be formed with the same diquarks of $X(3872)$ and $Z_{c}(3900)$, but seems too light to be the (radial) excitation of $Z_{c}$. Moreover, another whole family of tetraquarks generated by $Z_{c}^{\prime}$ should be expected, making this interpretation unfavored for the moment.

However, it is not clear why $Z_{c}^{\prime}$ has not been seen in Fig. 2: in a $1^{+-} D^{*} D^{*}$ molecule the $c \bar{c}$ pair contains both $S=0$ and $S=1$ components, and the $S$-wave decays into $J / \psi \pi$ and $\eta_{c} \rho$ should be favored with respect to the $P$-wave decay into $h_{c} \pi$. The question is still open.

\section{Exotic states as Feshbach molecules}

All quantitative evaluations of molecular spectra push the states at- or below-threshold (e.g. see Ref. [18] or the loop contribution in Ref. [16]). As we said, the mechanism for which some molecules are pushed above threshold is still unclear. To explain this, we proposed in Ref. [19] a temptative description in terms of Feshbach resonances.

We consider that the $X$ and $Z$ resonances are 4-quark states with flavor content $c \bar{c} q \bar{q}$. The color structure is free to rearrange in two ways: i) a hadrocharmonium (hadrobottomomium) [9] configuration where the $c \bar{c}$ pair is surrounded by light quak matter, $i i)$ an open charm configuration of two $D$ mesons. The mass eigenstate is a superposition of these two configurations ${ }^{3}$. We call $P$ and $Q$ respectively the open charm subspace and the hadrocharmonium (closed) subspace.

We assume that the hadrocharmonium system admits bound states giving rise to a discrete spectrum of levels. We roughly expect these levels to be in correspondence of the sum of the masses of hadrocharmonium 'constituents'. The coupling between the $P$ and $Q$ subspaces, described by some $H_{Q P}$ Hamiltonian term, appears in the expression of the scattering length

$a \simeq a_{P}+C \sum_{n} \frac{\left|\left\langle\Psi_{n}\left|H_{Q P}\right| \Psi_{\mathrm{th}}\right\rangle\right|^{2}}{E_{\mathrm{th}}-E_{n}} \simeq a_{N R}-C \frac{\left|\left\langle\Psi_{\mathrm{res}}\left|H_{Q P}\right| \Psi_{\mathrm{th}}\right\rangle\right|^{2}}{v}$

If an hadrocharmonium level in the $Q$ subspace happens to be close and above the onset of the continuum spectrum of levels in the $P$ subspace, we can neglect all but this term in the sum, giving rise to an attractive interaction and favoring the formation of a metastable resonance at the hadrocharmonium level, that is called Feshbach resonance (Fig. 3). The effect is enhanced the smaller the difference in energy $E-E_{\mathrm{th}}=v, E$ being the bound state level and $E_{\mathrm{th}}$ the open-charm threshold energy [20]. The Feshbach phe-

\footnotetext{
${ }^{3}$ This reflects the fact that, from a QFT point of view, the interpolating operators of molecules and hadrocharmonium have the same quantum number, and mix under renormalization.
}

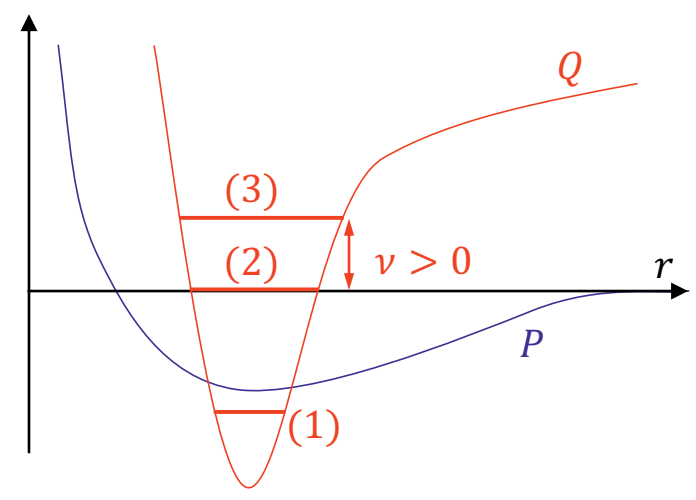

Figure 3. $P$ and $Q$ are the open and closed channels respectively. (1) Charged $X$ (3872) partners suppression, (2) $X$ case (3), $Z_{c, b}$ case.

\footnotetext{
${ }^{2}$ In this case, the virtual photon would decay into $Z_{c}^{\prime} \pi$ in $P$-wave.
} 


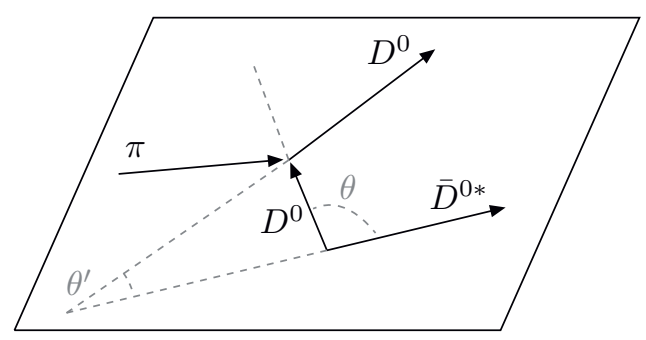

Figure 4. The elastic scattering of a $D^{0}$ (or $D^{* 0}$ ) with a pion among those produced in hadronization could reduce the relative momentum $\boldsymbol{k}_{0}$ in the centre of mass of the $D^{0} \bar{D}^{0 *}$ pair.

nomenon is therefore the formation of a resonance in the 'scattering' between different internal tetraquark states.

As for the $X(3872)$, we can consider an hadrocharmonium level with a $c \bar{c}$ pair with the quantum numbers of the $J / \psi$ and a light component with the quantum numbers of the $\rho^{0}$, held together by hadronic Van der Waalslike forces. This ' $J / \psi \rho \rho^{0}$, level would have a mass of $3872 \mathrm{MeV}$. The open charm threshold is at $3872 \mathrm{MeV}$ as well, so that Feshbach mechanism give rise to a huge scattering length, and to a very narrow state.

As for charged isospin partner of the $X(3872)$, we must consider that the charged threshold $D^{+} \bar{D}^{* 0}$ is at $\approx 3876$ $\mathrm{MeV}$, whereas the ' $\mathrm{J} / \psi \rho^{+}$' level is still at $3872 \mathrm{MeV}$. The level is now below threshold, so that $v<0$. The scattering length $a$ becomes positive because of the term $\propto 1 / v$. This is the signal that the interaction is repulsive, thus destabilizing the system. This mechanism implies the absence of charged partners and the occurrence of isospin violation.

We can extend this argument to $Z_{c}$ s and $Z_{b}$ s states. The decay width of a Feshbach resonance can be extimated to be $\Gamma=A \sqrt{v}$ [20]. We report in Tab. 2 the results of a global fit with existing data, reporting some interesting agreement with data. In particular, the constant $A$ is compatible to be the same for all states, both in charm and bottom sectors.

The $1^{--} Y(4260)$ cannot be described in a similar way. The closed charm state could be the $\psi(2 S) \sigma$, but the state would fall more than $30 \mathrm{MeV}$ below the $D_{1} \overline{D^{0}}$ threshold, thus making Feshbach formalism not applicable. However, even if considered a real $D D_{1}$ molecule, such a large binding energy would make the molecule tightly bound and long-lived, but $\Gamma_{Y(4260)} \gtrsim 100 \mathrm{MeV}$. Some authors have proposed the $Y(4260)$ to be an hybrid meson [8], which does not appear in spectra of purely multiquark interpolating operators, as hadrocharmonium or molecules. If confirmed, this hypothesis could accomodate this missing part.

\section{Prompt production of $X(3872)$ at hadron colliders}

In this section we focus on the problem of understanding prompt ${ }^{4}$ production of $X(3872)$ at hadron colliders. In Ref. [21] a question was raised: how is that possible

\footnotetext{
${ }^{4}$ i.e. not produced in $B$ decays but at the hadron collision vertex.
}

\begin{tabular}{l|c|cc|cc} 
& & Herwig & & Pythia & \\
\hline$k_{0}^{\max }$ & & $50 \mathrm{MeV}$ & $100 \mathrm{MeV}$ & $50 \mathrm{MeV}$ & $100 \mathrm{MeV}$ \\
\hline $\mathrm{N}$ & $0 \pi$ & 52 & 253 & 240 & 1560 \\
& $1 \pi$ & 44 & 299 & 283 & 1984 \\
& $3 \pi$ & 843 & 2069 & 4843 & 11679 \\
& $4 \pi$ & 1166 & 2802 & 6489 & 14916 \\
& $5 \pi$ & 1689 & 4167 & 7770 & 18284 \\
\hline$\sigma$ & $0 \pi$ & 0.10 & 0.50 & 0.13 & 0.83 \\
$(\mathrm{nb})$ & $1 \pi$ & 0.09 & 0.59 & 0.15 & 1.05 \\
& $3 \pi$ & 1.67 & 4.10 & 2.57 & 6.20 \\
& $4 \pi$ & 2.31 & 5.55 & 3.44 & 7.92 \\
& $5 \pi$ & 3.34 & 8.25 & 4.12 & 9.71 \\
\hline
\end{tabular}

Table 3. Number of molecule candidates generated with Herwig and Pythia, with different estimates for $k_{0}^{\max }$ and different number of scattering events with pions.

that a very long-lived molecule of a $D^{0}$ and a $D^{* 0}$ meson, with binding energy compatible with zero, could be formed within the hadrons ejected in $p \bar{p}(p)$ collisions at energies of some TeV? More specifically, the binding energy $\mathcal{E}_{b}=M_{X(3872)}-M_{D^{0}}-M_{D^{* 0}} \simeq-0.14 \mathrm{MeV}$ and a single well model were used to estimate the mean squared momentum $k_{0}^{\max } \simeq 50 \mathrm{MeV}$. This number can be used as an upper bound for relative momentum in center of mass frame of $D^{0} \bar{D}^{* 0}$ pairs, so as to be eligible candidates for becoming molecular loosely bound states. The authors of Ref. [21] performed numerical simulations with standard hadronization algorithms (Herwig and Pythia), simulated production of open charm mesons and selected the $D D^{*}$ pairs with a relative momentum as small as $k_{0}^{\max }$, and finally estimated an upper bound for the prompt production cross section of the $X(3872)$ which was found to be $\sim 300$ times smaller than the experimental value, thus challenging the molecular interpretation of $X(3872)$.

Such a gap did not seem to be unbridgeable to the authors of [22], who resorted to final state interaction (FSI) mechanisms in the $D^{0} \bar{D}^{* 0}$ system in order to improve the theoretical cross section up to the experimental value. In particular, it was suggested that FSI allow the $D D^{*}$ pairs to get $k_{0}^{\max } \sim 300 \mathrm{MeV}$ without breaking the bound state. This approach was criticised in [23], by noting that the presence of a huge numbers of pions between the $D D^{*}$ mesons make FSI approach unjustified. The controversy remained somewhat unsolved [24] ${ }^{5}$.

In Ref. [26] a somewhat opposite mechanism was suggested. It was considered that some of the large number of pions produced in the neighbourhood of the open charm meson pairs could scatter elastically on the $D^{0}$ or $D^{* 0}$ component of the would-be-molecule, thus changing the relative momentum in the centre of mass of the pair, $\boldsymbol{k}_{0}$ (Fig. 4). As discussed in [21], the spectrum of $D^{0} \bar{D}^{* 0}$ pairs before tha interaction with pions can be represented by a monotonically rising histogram in $k_{0}$. If even a small part of them was rearranged within lower relative momenta,

${ }^{5}$ FSI have recently been used to estimate $Z_{c, b}$ prompt production cross-sections at hadron colliders [25]. However, the application of this formalism to above-threshold states is unclear and somewhat arbitrary. 

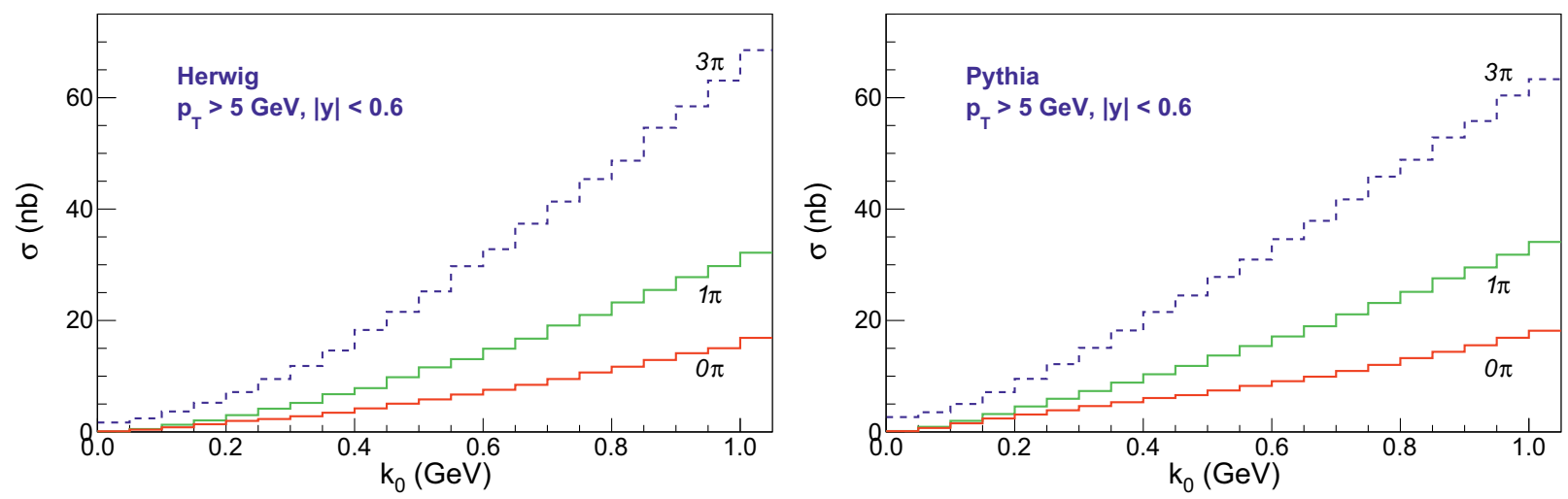

Figure 5. Number of $D^{0} \bar{D}^{* 0}$ pairs (events) counted with Herwig (left panel) and Pythia (right panel) when generating resp. $9 \times 10^{10}$ and $5 \times 10^{10}$ full QCD events at $\sqrt{s}=1.96 \mathrm{TeV}$ with the cuts on partons and hadrons described in Ref. [26]. The histograms named $1 \pi$ and $3 \pi$ are related to the elastic scattering of open charm mesons with one or three pions selected.

there could be a significant effect of feed-down of pairs towards lower bins, even in the far low energy region below $50 \mathrm{MeV}$. Populating that region means increasing the formation probability of the loosely bound $X$.

In Ref. [26] a first qualitative exploration of this phenomenon was performed, by generating samples of $p \bar{p} \rightarrow$ $c \bar{c}(X)$ events in Herwig and Pythia, at Tevatron COM energies $\sqrt{s}=1.96 \mathrm{TeV}$. Then, a pion in proximity of one of the mesons is selected, the elastic interaction (regulated by effective matrix element) is implemented. It was noticed that the number of pairs with $k_{0}<k_{0}^{\max }$ was sensibly increased.

Furthermore, the open charm mesons might interact with pions $2 \div 3$ times before a molecule is formed. Hence the $k_{0}^{(n)}$ after $n$ interactions was evaluated by assuming the probability distribution function which regulates the variation of $k_{0}$ to be the same for all the interactions, like in a Markov chain. The results showed a striking increase by a factor of $\sim 100$ of the $D D^{*}$ molecule candidates, thus qualitatively indicating that the mechanism might play an important role in physical events.

We present here preliminary results of the full QCD events $p \bar{p} \rightarrow c \bar{c}, p \bar{p} \rightarrow g g, p \bar{p} \rightarrow g q, p \bar{p} \rightarrow q q \ldots$ We generated $9 \times 10^{10}$ events in Herwig and $5 \times 10^{10}$ events in Pythia. With full QCD, we noticed that more than $95 \%$ of $c \bar{c}$ pairs are produced during the parton shower process by softer and softer gluons, so that the number of low-relative-momentum $D D^{*}$ pairs is larger with respect to $p \bar{p} \rightarrow c \bar{c}$ events. Following the same analysis as in Ref. [26], and get the results reported in Fig. 5 and Tab. 3. We see that with full QCD the enhancement due to interactions with pions is not so relevant as in $c \bar{c}$ generation. In fact, we increase the cross section only by a factor $O(10)$, instead that $O(100)$ as in [26]. If we take into account a $k_{0}^{\max }=100 \mathrm{MeV}$, and if we consider $3 \div 5$ interactions with pions, we can get only up to $30 \%$ of experimental cross section. We remark that also Refs. [22, 25] take into account only events $p \bar{p} \rightarrow g c \bar{c}$, and a full QCD analysis is needed also with FSI. Finally, a deeper analysis of the effect of such mechanisms on known differential cross section distributions is ongoing.

\section{Doubly charmed states}

The last topic we deal with is another approach to choose among possible exotic models. We propose indeed to predict exotic states which fit only one model, and have neat experimental signatures. In particular, in Ref. [27] we proposed to look for doubly charmed tetraquark states of the form $[c c]\left[\bar{q}_{1} \bar{q}_{2}\right]$, with $q_{1}, q_{2}=u, d, s$, that we call $\mathcal{T}_{(s, s s)}^{0,+++}$. In particular, the doubly charged states obtained with $q_{1}, q_{2}=d, s$ could not be interpreted in terms of loosely bound molecules $D^{+} D^{+}$even if they occurred at threshold, because of the Coulomb repulsion.

In Ref. [27] the phenomenology of such states was investigated according to the diquark-antidiquark picture, and decay widths and production branching fractions from $B_{c}$ mesons and $\Xi_{b c}$ baryons were estimated (Fig. 6).

Furthermore, from a theoretical point of view, the flavor quantum numbers of the $\mathcal{T}$ give the possibility to clearly distinguish these tetraquarks from conventional quarkonia. A method by Lüscher [28] established a connection between the two-particle discrete eigenvalues of the finite volume QCD Hamiltonian and the matrix elements of the infinite volume scattering matrix under the inelastic threshold, thus allowing lattice simulations to make predictions on QCD unstable states. A lattice simulation is currently ongoing.

\section{Acknowledgments}

I wish to thank G. Montagna and the conveners for their invitation. I also thank F. Piccinini and A. D. Polosa for their continuous support.

\section{References}

[1] S. F. Radford and W. W. Repko, Phys. Rev. D 75, 074031 (2007) [hep-ph/0701117]. 

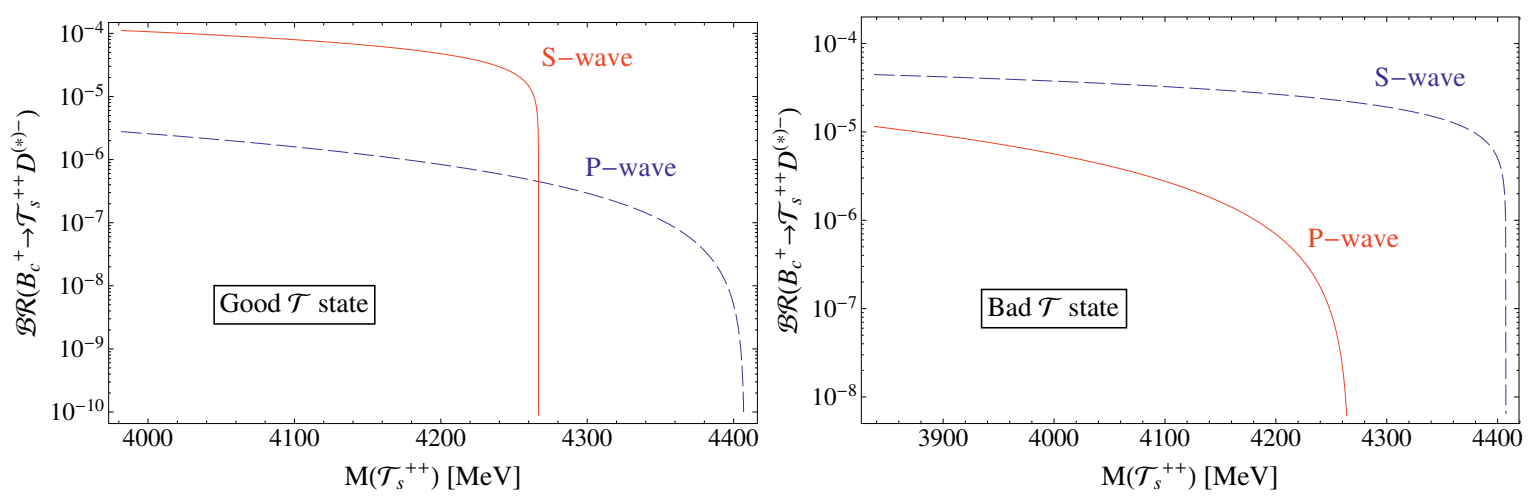

Figure 6. Branching ratios calculated in [27] for the production of $B_{c}^{+} \rightarrow \mathcal{T}_{s}^{++} D^{-}$(blue) and $B_{c}^{+} \rightarrow \mathcal{T}_{s}^{++} D^{*-}$ (red) in the hypothesis of quantum numbers $J^{P}=1^{+}$(left panel) and $0^{+}$(right panel) as a function of the mass of $\mathcal{T}_{s}^{++}$, in the above-threshold region.

[2] G. T. Bodwin, E. Braaten and G. P. Lepage, Phys. Rev. D 51, 1125 (1995) [Erratum-ibid. D 55, 5853 (1997)] [hep-ph/9407339].

[3] N. A. Tornqvist, Z. Phys. C 61, 525 (1994) [hepph/9310247]; Phys. Lett. B 590, 209 (2004) [hepph/0402237]; E. S. Swanson, Phys. Rept. 429, 243 (2006) [hep-ph/0601110];

[4] M. P. Valderrama, Phys. Rev. D 85, 114037 (2012) [arXiv:1204.2400 [hep-ph]].

[5] E. Braaten and M. Kusunoki, Phys. Rev. D 69, 074005 (2004) [hep-ph/0311147].

[6] R. L. Jaffe, Phys. Rept. 409, 1 (2005) [hep$\mathrm{ph} / 0409065]$.

[7] L. Maiani, F. Piccinini, A. D. Polosa and V. Riquer, Phys. Rev. D 71, 014028 (2005) [hep-ph/0412098];

[8] L. Liu et al. [Hadron Spectrum Collaboration], JHEP 1207, 126 (2012) [arXiv:1204.5425 [hep-ph]]; E. Kou and O. Pene, Phys.Lett. B631 (2005) 164-169, [hepph/0507119]; P. Guo, A. P. Szczepaniak, G. Galata, A. Vassallo and E. Santopinto, Phys. Rev. D 78, 056003 (2008) [arXiv:0807.2721 [hep-ph]].

[9] S. Dubynskiy and M. B. Voloshin, Phys. Lett. B 666, 344 (2008) [arXiv:0803.2224 [hep-ph]].

[10] M. Ablikim et al. [BESIII Collaboration], Phys. Rev. Lett. 110, 252001 (2013) [arXiv:1303.5949 [hep-ex]]; Z. Q. Liu et al. [Belle Collaboration], Phys. Rev. Lett. 110, 252002 (2013) [arXiv:1304.0121 [hep-ex]].

[11] M. Ablikim et al. [BESIII Collaboration], arXiv:1310.1163 [hep-ex].

[12] M. Ablikim et al. [BESIII Collaboration], arXiv:1308.2760 [hep-ex]; arXiv:1309.1896 [hepex].

[13] A. Bondar et al. [Belle Collaboration], Phys. Rev. Lett. 108, 122001 (2012) [arXiv:1110.2251 [hep-ex]].

[14] R. Faccini, A. Pilloni and A. D. Polosa, Mod. Phys. Lett. A 27, 1230025 (2012) [arXiv:1209.0107 [hep$\mathrm{ph}]$.
[15] T. Xiao, S. Dobbs, A. Tomaradze and K. K. Seth, Phys. Lett. B 727, 366 (2013) [arXiv:1304.3036 [hepex]]

[16] Q. Wang, C. Hanhart and Q. Zhao, Phys. Rev. Lett. 111, 132003 (2013) [arXiv:1303.6355 [hep-ph]].

[17] R. Faccini, L. Maiani, F. Piccinini, A. Pilloni, A. D. Polosa and V. Riquer, Phys. Rev. D 87, 111102 (2013) [arXiv:1303.6857 [hep-ph]];

[18] F. -K. Guo, C. Hidalgo-Duque, J. Nieves and M. P. Valderrama, Phys. Rev. D 88, 054014 (2013) [arXiv:1305.4052 [hep-ph]].

[19] M. Papinutto, F. Piccinini, A. Pilloni, A. D. Polosa and N. Tantalo, arXiv:1311.7374 [hep-ph].

[20] A. J. Leggett, Quantum Liquids, Oxford 2006; C. J. Pethick and H. Smith, Bose-Einstein Condensation in Dilute Gases, Cambridge 2008.

[21] C. Bignamini, B. Grinstein, F. Piccinini, A.D. Polosa and C. Sabelli, Phys. Rev. Lett. 103, 162001 (2009) [arXiv:0906.0882 [hep-ph]].

[22] P. Artoisenet and E. Braaten, Phys. Rev. D 81, 114018 (2010) [arXiv:0911.2016 [hep-ph]].

[23] C. Bignamini et al.,Phys. Lett. B 684, 228 (2010) [arXiv:0912.5064 [hep-ph]].

[24] P. Artoisenet and E. Braaten, Phys. Rev. D 83, 014019 (2011) [arXiv:1007.2868 [hep-ph]].

[25] F. K. Guo, U. G. Meißner and W. Wang, arXiv:1308.0193 [hep-ph].

[26] A. Esposito, F. Piccinini, A. Pilloni and A. D. Polosa, J. Mod. Phys. 4, 1569 (2013) [arXiv:1305.0527 [hep$\mathrm{ph}]$.

[27] A. Esposito, M. Papinutto, A. Pilloni, A. D. Polosa and N. Tantalo, Phys. Rev. D 88, 054029 (2013) [arXiv:1307.2873 [hep-ph]].

[28] M. Luscher, Commun. Math. Phys. 105 (1986) 153; Nucl. Phys. B 354 (1991) 531; Nucl. Phys. B 364 (1991) 237. 